and fresh, is a neutral fluid, is eminently grateful to the human stomach in infancy; but as regards adults, the case seems somewhat to alter. It may be that milk is not intended to be used by adult animals, for thereby the supply for the young would be unduly diminished; and this is not the only economical provision of a similar kind in nature which we may observe. When milk does create stomach disturbance in an adult, it is generally to the butyric constituent that we trace the derangement in question, which consists of rancid eructations \&.c.; and we wonder that an ingredient, which forms only from two and a half to three and a half per cent. of the integral fluid, should be the source of those often severe symptoms which we witness. Possibly the caseine, coagulated by the stomachic acids into curd, may contribute to the morbid effects just referred to.

While, however, milk disagrees, in the manner described, with many adults, in others it causes very different, but not less formidable, derangements. It appears to exert a singularly sedative effect on the duodenum, the condition of which portion of the intestinal canal seems (by sympatly) to regulate the degree of activity of the hepatic secretion. Under the influence of milk, used largely by an adult not accustomed to it, the biliary secretion, or at least the excretion of bile, seems to become nearly or wholly suspended, the liver tumefies, and a mild form of jaundice ensues; the eyes, and even the skin, assume a yellow hue; the tongne is coated with a yellow fur; the stools are pale; and that drowsiness of body and apathy of mind which characterize idiopathic icterus, are experienced in an incipient degree. Popular wisdom explains all this, by saying curtly that milk is bilious. But milk contains less of the materials of bile than many other kinds of food. The true explanation is, that to the infantile stomach, which has never known any other alinent, or the artificial stimulation of our various saline, acidulous, pungent, peppery condiments, milk is an article which sufficiently ex. cites the unsophisticated digestive sensibilities. But when an adult, accustomed to the artificial stimulants above described, suddenly resorts, from a freak of his own, or that of an indiscreet medical adviser, to a large use of milk, with a view to mitigate some dyspeptic irritation, he soon experiences the untoward effects which we have above enumerated, and which arise from too little stimulation of the duodenum and orifice of the ductus communis.

There are various modes of avoiding these effects-I. We may dilute the milk with water. 2. Boiling the milk seems to lessen the chance of its deranging the stomach. 3. Adding a little brandy to it has the same effect, and is perluaps the most eligible amendment.

Rayenscourt-park Villas, Hammersmith.

\section{ON DUMB-BELL CRYSTALS OF URIC ACID DISCOVERED IN THE URINE.}

\section{By MARRIS WILSON, M.D.}

THE most remarkable and curious of all the formations which occur in urine is the dumb-bell, attention to which was first drawn by Dr. Golding Bird, by whom it was described as a condition peculiar to oxalate of lime. More recently, Dr. Hassall (see The LANoet, vol. i. 1850, p. 177) has pointed it out as a form assumed by other oxalates, and also by soda and potash, in combination with sulphuric acid.

Having been for some time past engaged in the analysis of urine, in a variety of diseases, I was led to make a series of experiments, for the purpose of determining the condition of the urine favourable to this formation; and in the course of these observations was enabled to determine the following interesting facts:-

Firstly. That dumb-bell crystals are very frequently developed, as the result of certain modifications of uric acid.

Secondly. That it is not to the salts of urine alone that this formation is confined, for $I$ have frequently met with it in solutions which had no organic origin, and in a variety of salts.

I should perhaps premise, that although Nature seems to accomplish her ends without effort or excess, and sometimes most unexpectedly, it is occasionaily very difficult, in prosecuting experiments, to arrive at satisfactory results. I am led however, to the conclusion, that the form of the dumb-bell crystal depends upon a specific but independent force, developed under certain determinate circumstances, consentaneously with that of homogeneous attraction or aggregation.

I have observed, that when spicular crystals commence forming upon a centre, after stretching some little distance in a straight line, they begin to branch and spread outwards; and that as this lateral force is more or less vigorous, that of extension is arrested, and the tuft of spiculæ spreading from the central point, assumes the flattened globular shape, in a proportionately complete degree, and the same action taking place at both ends of the primary crystal, constitutes the dumb-bell form. It appears to me that this effect arises purely from the agency of electricity; and that, as an arranging power, it is quite separate and distinct from that of aggregation, and depends for its influence upon the repulsive force manifested towards each other by similarly electrified bodies.

The first effect of consolidation in the crystalline centre is the production of a small amount of electrical action, suffi. cient in itself to cause two especial points of that centre when it is the basis of a future dumb-bell, to assume a mutual repulsion.

This compound formative power, being directed on two opposite points of the mass, will continue, until arrested by consolidation.

As the electrical or arranging force predominates, a limit will be fixed to the longitudinal extension of the crystals and proportionately the lateral arrangement propagated, until a more or less complete dumb-bell is produced.

Occasionally this latter action is so powerful as to fill up with spicula and consolidate the entire space around the centre of crystallization.

But it is not my intention to enter into the very interesting subject of crystallization generally, many new phenomena of which actual examination by the microscope has taught me, but to confine myself to the form and construction of the dumb-beils of uric acid. Uric acid and oxalate of lime occur very frequently together in the same deposit, and from their combined presence very different forms of dumb-bell crystals are found together; it is therefure of the first importance to separate each salt carefully, before proceeding to subject it to experiment.

While analyzing some urine of high specific gravity, and in which there was a copious precipitate of uric acid I added a solution of caustic potash in sufficient quantity to dissolve the deposit. The urine thus treated was twice filtered; a light flocculent cloud of uric acid, in combination with potash, made its appearance in a short time, and gradually subsided to the bottom of the vessel. The supernatant fiuid being decanted off, hydrochloric acid, in great excess, was poured suddenly upon the sediment, which, after a rest of about a quarter of an hour, was found converted into a thin layer of opake dark. brown uric acid dumb-bells. These differed in the most marked degree from the dumb-bell of oxalic origin by their great in solubility in water. But the experiment is open to the doubt that an oxalate might have gained entrance.

I then separated some highly crystallized uric acid from the urine in which it was deposited, and washed it thoroughly with large quantities of warm water; it was then dissolved in pure caustic potash, and filtered through paper. On adding strong hydrochloric acid in excess to this solution, dumb-bell crystals were deposited, some being of very perfect form, others not so.

Some pure lithic acid was dissolved in concentrated sulphuric acid; to this solution two-thirds of water were added, and there occurred an instantaneous precipitation of imperfect dumb-bells. To show the difficulties of this kind of demonstration, and the changes likely to result from varied circumstances, a small quantity of this concentrated sulphuric solution being dropped into a large bulk of water, the resulting deposit consisted of thin broad plates, the two edges of which, in the longitudinal direction of the spiculæ, were considerably thickened, forming ridges; but these plates, when rolled over on their thin edges for examination, also presented a dumbbell form, though from their narrowness it was impossible to retain them on the edge.

The dumb-bells to which I wish to call attention and give a description are those obtained in the deposit caused by the addition of hydrochloric acid to the potash solution. My reason for so doing is because $I$ have observed the same forms as a natural deposit among uric acid; and these, although of such a remarkable form, have, I believe, until the present time, been left undescribed.

They consist of a thin translucent plate, of an oval or rhomboid form, having a slightly elevated ridge running along their middle in the direction of the long diameter, and from near the extremities of which extend two cornpact kidney-shaped crests of thin spicular crystals, their long diameter being across the plane of the translucent plate. A transverse section of one of the kidney-shaped crests would present an oval outline. 
From the way in which the weight is arranged-namely, in the centre of the crests-the crystal has, while under the microscope, a tendency to float with the edge of the thin plate turned towards the eye of the observer. Circumstances, however, occasionally seem to dispose the face of the thin plate to be directed upwards, and then, without a close and careful examination by rolling the crystals over, their dumbbell shapes might escape the eye of the microscopist, and seem to represent only rhomboid plates of uric acid.

In the hydrochloric deposit there was considerable variety of size, the following being measurements made with one of Powell's large microscopes:-

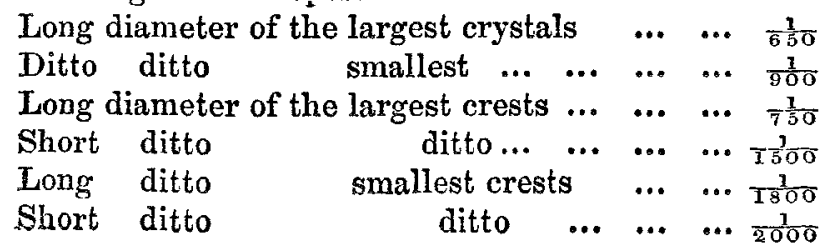

Fia. 1.

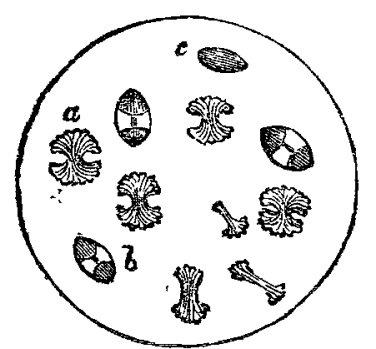

Represents the dumb-bell crystal as deposited by the addition of hydrochloric acid to the potash solution of uric acid.

a. The crystal with the edge of the thin plate turned towards the eve of the observer.

$b$. The same rolled over to show the face of the thin plate.

c. Shape of a transverse section of a kidney-shaped crest.

Fia. 2.

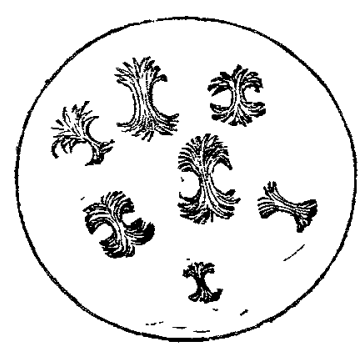

The same shapes deposited from a concentrated sulphuric solution.

Frg. 3.

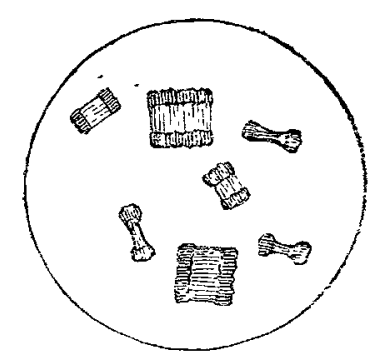

The forms assumed by the crystal when a small quantity of the sulphuric solution is dropped into a large quantity of water.

The Cholera. - At Vienna the disease was raging with much severity on the 7th of September, particularly among the labourers employed on the Scmmering Railroad. The disease is on the increase at Pesth, and has almost de. populated a small town of Moravia, called Grosshreflein. Sixty-eight persons died in two days, being five per cent. of the population, which is but thirteen hundred. The numbers returned at Vienna, since the reappearance of the disease, are 1019 cases; ont of which there have been 432 recoveries, 422 deaths, and 165 persons are still under treatment. The cholera had disappeared from Mexico on the 13th of August: the disease reigned exactly one hundred days, during which time no less than 1800 persons perished.

\section{A Glance at the Entrodutoty 垈ectures \\ DELIVERED IN THE}

\section{IONDON AND PROVINCIAL SCHOOLS OE MEDICINE,}

AT THE OPENTIG OF THE SESSION 1850.

\section{KING'S COLLEGE.}

Abstract of the Introductory Lecture of Professor L. Forbes.

THE studies which engage the attention and occupy the time of the medical student may be classified under three heads: human and general anatomy, physiology, pathology, and materia medica, laying a sound foundation of accurateand indispensable knowledge; the theory of medicine and surgery, acquired in the lecture room, and an initiation into practice by the bedside of the patient; the theory and practice of midwifery completing his purely professional acquirements. But to form. an accomplished physician or surgeon, and to complete a sound medical education, there is another class of studies, represented by chemistry, botany, zoology, and comparative anatomy. Each of these sections of science has, it is true, a direct and practical bearing upon medicine, but that is of little value, compared with their educational importance, as means of training the observing faculties of the student. The advantage in " training the faculties, \&c.," is the ground of apology for introducing mathematics, algebra, \&c., into the preliminary studies of medical aspirants. Comparative anatomy, botany, and chemistry, call for no apology on such a ground. On correct observation, a careful induction to successful pursuit of medical science must be founded. Those who look only to results and final purposes are apt to underrate the value of a minute training of the observing powers. Just as in ordinary education, classical and mathematical studies serve to exercise, strengthen, and develop the mind, so as to enable it, in whatever direction its thoughts may eventually be turned, to meet the pursuits of life, and grapple with its difficulties with vigour and understanding; so do the sciences of chemistry and natural history prepare the student of medicine, whatever may be the line of practice to which he may eventually direct his attention, to observe the phenomena of disease with minuteness and accuracy, and to form rapid and just conclusions respecting their origin and progress. All the chemistry in the world, and all the natural history, will not make a man a good physician, regarded merely as so much knowledge in those sciences; but a practical acquaintance with the methods of observation in chemistry, dealing as they do so much with the sequence of phenomena, and in natural history, regarding, as they do, the exact definition of the object, cannot fail to benefit the stndent of medicine, and to give him power peculiarly applicable to the successful pursuit of his profession.

That men are naturally, and uninstructed, bad observers, we see every day in the attempts of travellers to describs animated objects. Without such a training in observation as a course of medical education gives, their descriptions are false and useless, unless the describers have been previously versed in natural history. Even among honest and earnest votaries of science, who have either entered upon its pursuit late in life, and have attempted original inquiry without a sufficient know. ledge of its rudiments, or who have engaged in complicated researches, without a previous training in the methods of $\mathrm{ob}$ servation, we are too often forced to listen to absurdities, visionary speculations based upon ill-observed facts, or fantastic misapprehensions of the facts themselves. This is especially the case with the crowd of uninstructed microscopic observers, who daily enter inconsiderately on abstruse inquiries, which demand, ere they can be successfully investigated, the sound foundation of a physiological education, of which correct observation is an essential element. The best instrument is only a source of deception unless there be a trained eye, and a sound head at the end of it. When every day we are reminded by what we hear and read, that without training few if any men are accurate observers,-a fact especially conspicuous in a country like ours, where the training of the observing powers is too often systematically neglected in the education of youth, - how important does it become that in the preparation for the practice of the medical profession, every means should be taken to form the student into a good observer! In the practice of medicine, defective powers of observation, or incapability, through defective training, of noting accurately the phenomena of disease, must inevitably endanger alike the life of the patient, and the reputation of the practitioner. On one mistake in the observation of a fact, a long train of false reasoning may be founded, pernicious 